\title{
Lupus eritematoso sistémico, en un paciente varón: a propósito de un caso
}

\author{
Systemic lupus erythematosus in a male patient: presentation of a case
}

\author{
Franco Romaní ${ }^{1}$, Fernando Atencia ${ }^{2}$, José Cuadra ${ }^{3}$, Carlos Canelo ${ }^{3}$ \\ ${ }^{1}$ Interno de Medicina, Hospital Nacional Arzobispo Loayza, Facultad de Medicina de San Fernando, UNMSM. \\ ${ }^{2}$ Médico Internista Asistente, Hospital Nacional Arzobispo Loayza, Docente de la Facultad de Medicina de San Fernando, UNMSM. \\ ${ }^{3}$ Médico Residente de Medicina Interna, Hospital Nacional Arzobispo Loayza, UNMSM-
}

\section{Resumen}

El lupus eritematoso sistémico es una enfermedad que afecta predominantemente a mujeres, presentándose con manifestaciones variables. Esta comunicación de caso trata sobre un paciente varón joven, que debuta con artropatía lúpica, variedad artropatía de Jaccoud. Se realiza una revisión de la literatura acerca de las características clínicas del lupus en el varón y sus diferencias con el cuadro clínico en la mujer.

Palabras clave: Lupus eritematoso sistémico; masculino; artropatías.

\begin{abstract}
Systemic lupus erythematosus is a disease that affects women predominantly, with variable manifestations. This is a case report on a young male patient that debuts with lupus arthropathy, variety Jaccoud's arthropathy. We review the clinical characteristics of lupus in males and the differences with the disease in females.
\end{abstract}

Key words: Lupus erythematosus, systemic; male; joint diseases.

\section{INTRODUCCIÓN}

El lupus eritematoso sistémico (LES) es una enfermedad mutisistémica autoinmune, que afecta a mujeres casi en $90 \%$ de todos los casos; la incidencia en varones varia de $4 \%$ a $22 \%$, según distintas series, por lo que la proporción mujer/varón es de aproximadamente 9 a 1. A continuación, presentamos el caso de un varón que debutó con compromiso articular, fenómeno de Raynaud, vasculitis cutánea y mononeuritis múltiple. Además, se realiza una discusión a propósito del caso, sobre las características clínicas e inmunológicas del LES en el paciente varón, en base a literatura mundial y nacional.

\section{REPORTE DE CASO}

Paciente varón de 18 años, mestizo, natural y procedente de Andahuaylas, previamente sano, sin antecedentes patológicos, personales y familiares de importancia. Inició su cuadro clínico 8 meses antes del ingreso, con dolor y tumefacción en ambas rodillas, que se intensificaban luego de actividad física. Seis meses antes del ingreso a hospitalización, se agregó limitación funcional de ambas rodillas, dolor y tumefacción en ambos tobillos y en articulación metatarsofalángica (MTF), que ocasionaban limitación y dificultad para la marcha. Cinco meses antes del ingreso, aparecieron dolor, tumefacción y limitación funcional en las articulaciones metacarpofalángicas (MCF), interfalángicas proximales (IFP), muñeca y codo, siendo estos síntomas más intensos por las mañanas y durando de $60 \mathrm{a}$ 90 minutos. El paciente además presentaba compromiso de columna cervical, con dolor y limitación funcional a la flexión, extensión y rotación lateral. Cuatro meses antes del ingreso, las artralgias se intensificaron, llevando al paciente a la postración en cama, motivo por el cual es internado en el hospital de origen; durante su hospitalización persistió con limitación funcional, dolor y tumefacción de articulaciones, dándosele de alta con leve mejoría. Se le agregó luego sensación de alza térmica no cuantificada, que se mantuvo en forma remitente; cinco días antes del ingreso se agregaron a los síntomas descritos edemas de ambos miembros inferiores, con lesiones purpúricas palpables en tercio inferior de pierna y parte dorsal del pie.

Al ingreso refería hiporexia y disminución ponderal de $10 \mathrm{~kg}$ en los últimos 4 meses. Las funciones vitales fueron: frecuencia cardiaca de 90 latidos por minuto, 22 respiraciones por minuto, presión arterial de 100/60 $\mathrm{mmHg}$, peso de $52 \mathrm{~kg}$ e índice de masa corporal de $18,42 \mathrm{~kg} / \mathrm{m} 2$. Ectoscópicamente, el paciente no tenía facies característica, estaba hidratado y adelgazado, colaborando con el examen. Piel pálida, lecho ungueal con cianosis y frialdad acral, además de lesiones purpúricas palpables en tercio inferior de ambas piernas, con leve edema en tercio inferior de pierna derecha.
En mucosa bucal, presentaba lesiones ulceradas no dolorosas. Se le palpaba ganglios linfáticos de $1,5 \mathrm{~cm}$ x $1 \mathrm{~cm}$, móviles, de consistencia aumentada, no pétreos, no dolorosos, en axilas, ingles y epitrócleas. Al examen del sistema osteomioarticular, se encontró dolor, tumefacción de partes blandas y limitación funcional bilateral, en articulaciones MTF, IFP proximales, muñecas, codos, MTF, tobillos y rodillas; además, se evidenció desviación cubital en ambas muñecas. En tórax y pulmones, el examen fue normal. Los ruidos cardiacos fueron rítmicos y de intensidad adecuada, con soplo holosistólico multifocal, pulsos periféricos presentes, sin ingurgitación yugular ni reflujo hepatoyugular. El abdomen fue blando y no doloroso, con hígado palpable a $5 \mathrm{~cm}$ por debajo del reborde costal derecho, con proyección hepática de $14 \mathrm{~cm}$, no se palpó punta de bazo. El examen neurológico no tuvo hallazgos significativos. Los exámenes auxiliares al ingreso fueron los siguientes: hematocrito $24 \%$, hemoglobina 7,9 g/dL, volumen corpuscular medio $81 \mathrm{fL}$, hemoglobina corpuscular media $27,1 \mathrm{pg}$, concentración media corpuscular de hemoglobina 33,3 g/dL y reticulocitos 2,3\%; leucocitos $9700 \mathrm{cel} / \mu \mathrm{L}$ (eosinófilos $8 \%$, abastonados $6 \%$, segmentados $65 \%$ y linfocitos $19 \%$ ); plaquetas $368000 \mathrm{cel} / \mu \mathrm{L}$; tiempo de protrombina 14,5 segundos y una razón normalizada internacional (INR, siglas en inglés) de 1,2. El frotis de sangre 
Tabla 1. Exámenes de orina durante la hospitalización.

\begin{tabular}{|c|c|c|c|c|c|}
\hline Variable & Primer control & Segundo control & Tercer control & Cuarto control & Quinto control \\
\hline Densidad & 1025 & 1024 & 1025 & 1030 & 1020 \\
\hline Proteínas & Trazas & $1+$ & $1+$ & $1+$ & $1+$ \\
\hline Hemoglobina & + & $2+$ & $3+++$ & $3+++$ & $2++$ \\
\hline Sangre oculta & + & $2+$ & $2++$ & $2+$ & \\
\hline \multicolumn{5}{|l|}{ Sedimento: } & 8-10 por campo \\
\hline Hematíes & $\begin{array}{l}35-40 \text { por campo } \\
(20 \% \text { crenocitos) }\end{array}$ & $\begin{array}{l}\text { 30-31 por campo } \\
\text { (100\% dismórficos) }\end{array}$ & $\begin{array}{l}80-90 \text { por campo } \\
\text { (80\% dismórficos) }\end{array}$ & $\begin{array}{l}\text { Abundantes por campo } \\
\text { (90\% dismórficos) }\end{array}$ & $\begin{array}{l}80-90 \text { por campo } \\
\text { (20\% dismórficos) }\end{array}$ \\
\hline Cilindros & $\begin{array}{l}\text { Cilindros granulosos } \\
2-3 \text { por campo. } \\
\text { Cilindros leucocitarios } \\
0-2 \text { por campo. }\end{array}$ & & & & \\
\hline $\begin{array}{l}\text { Depuración } \\
\text { de creatinina } \\
\text { en } 24 \text { horas } \\
(\mathrm{DH}: 6)\end{array}$ & & $\begin{array}{r}8 \\
\text { (Valor norr }\end{array}$ & $\begin{array}{l}5,5 \mathrm{~mL} / \mathrm{min} / 1,73 \mathrm{~m}^{2} \\
\text { nal: } 70-125 \mathrm{~mL} / \mathrm{min} / 1 \text {, }\end{array}$ & & \\
\hline $\begin{array}{l}\text { Proteinuria } \\
\text { en } 24 \text { horas }\end{array}$ & & & $1,70 \mathrm{~g} / 24$ horas & & \\
\hline
\end{tabular}

periférica reveló hipocromia $(+)$, anisocitosis $(+)$, autohemaglutinación $(++)$, leucocitos y plaquetas normales. En bioquímica, tuvo glicemia de $84 \mathrm{mg} / \mathrm{dL}$, urea $35 \mathrm{mg} / \mathrm{dL}$, creatinina 0,81 mg/dL, TGP 47 U/L, TGO 77 U/L, deshidrogenada láctica $460 \mathrm{U} / \mathrm{L}$ y bilirrubinas totales $0,21 \mathrm{mg} / \mathrm{dl}$. Se encontraron negativos los exámenes: gota gruesa, antígeno australiano, prueba rápida de $\mathrm{VIH} /$ sida y cinco coloraciones para bacilo de Koch en esputo. Los exámenes de orina se observa en la Tabla 1 . Se realizó exámenes auxiliares generales, inmunológicos y se descartó cuadros infecciosos, lo cual se detalla en la Tabla 2.

En cuanto al diagnóstico por imágenes, se realizó una ecografía abdominal superior, en la que se encontró hígado aumentado de tamaño $(21,8 \mathrm{~cm})$, con bordes uniformes, parénquima aumentado en ecogenicidad, sin tumoraciones ni colecciones, vena porta

Tabla 2. Exámenes generales e inmunológicos durante la hospitalización.

\begin{tabular}{lc}
\hline Variable & Resultados \\
\hline Parasitológico seriado & Negativo \\
Hemocultivo por tres & Negativo \\
VDRL & No reactivo \\
Urocultivo & Negativo \\
Coombs directo & Negativo \\
ANCA & Negativo \\
Factor reumatoide & Método de Waaler Rose: negativo \\
Rosa de bengala & Negativo \\
2 mercaptoetanol & Negativo \\
Ferritina & 40 Ul/mL: $18,7-323)$ \\
VSG & 318,77 ng/mL (V.N/hora \\
PCR & 2,58 mg/dL \\
Anticuerpos antinucleares & Positivo: Patrón moteado 1:640 (métofo IFA (HEP-2) \\
Anti ADN nativo & Positivo \\
Complemento C3 & 27 mg/dl \\
\hline
\end{tabular}

VDRL = Venereal Disease Research Laboratory test (Prueba de laboratorio para estudio de enfermedades venéreas).

ANCA = Anticuerpos contra el citoplasma de los neutrófilos.

VSG = Velocidad de sedimentación globular.

$\mathrm{PCR}=$ Proteína $\mathrm{C}$ reactiva

IFA = Detección de anticuerpos por Inmnunofluorescencia

HEP = Células Hep-2, nombre de células usadas para detectar anticuerpo antinucleares.

$\mathrm{ADN}=$ Acido desoxirribonucleico. de $14 \mathrm{~mm}$, bazo de consistencia homogénea y tamaño aumentado $(13,4 \mathrm{~cm})$. Las radiografías mostraron: en manos, discreta osteopenia yuxtaaricular, con pequeñas zonas de micronecrosis en extremos proximales de falanges proximales; en pelvis, sacroileítis bilateral, a predominio izquierdo. En tórax, los rayos $\mathrm{X}$ no mostraron hallazgos que contribuyeran. Se realizó ecocardiografía transtorácica, con función sistólica de ventrículo izquierdo normal (fracción de eyección: 78\%), morfología y función valvular normal.

El paciente fue sometido a electromiografía, en la que se encontró signos de mononeuritis múltiple y miopatía inflamatoria proximal. Por este motivo, se tomó biopsia de nervio sural, donde no se encontró evidencia de vasculitis. Durante la evolución, el paciente desarrolló lesiones purpúricas en miembros inferiores, tomándose biopsia de la piel afectada y se observó discreto engrosamiento de las paredes de los vasos de pequeño calibre, algunos con pequeños depósitos de mucina en su pared, extravasación de glóbulos rojos y mínimo componente inflamatorio perivascular, lo cual es compatible con vasculitis livedoide o vasculitis de vasos pequeños, limitada por intervención terapéutica.

Se le realizó el diagnóstico de lupus eritematoso sistémico, según los criterios diagnósticos utilizados por el Colegio Americano de Reumatología (Tabla
3), tras lo cual, y en vista de la evolución clínica, se realizó biopsia renal, en que se encontró compromiso por LES, descrita como glomerulonefritis proliferativa difusa (Tipo IV-a).

El paciente fue tratado con 3 pulsos intravenosos de $1 \mathrm{~g}$ de metilprednisolona y un pulso intravenoso de ciclofosfamida, a dosis de 0,5 g/m2 SC, tras los cual presentó mejoría clínica significativa y fue dado de alta, con tratamiento vía oral con prednisona, a dosis de $1,5 \mathrm{mg} / \mathrm{kg} /$ día.

\section{DISCUSIÓN}

El lupus eritematoso sistémico (LES) es una enfermedad inflamatoria multisistémica autoinmune, crónica, recurrente y potencialmente fatal ${ }^{(1,2)}$. Factores genéticos, ambientales y sociodemográficos tienen roles importantes en la patogénesis y expresión de la enfermedad. Esta multiplicidad de factores etiológicos podría explicar la variabilidad de manifestaciones observadas, no solo entre individuos sino también entre grupos étnicos ${ }^{(3)}$. Se sabe que las mujeres son nueve veces más afectadas que los varones, por lo cual se le ha considerado tradicionalmente como una enfermedad de mujeres. La marcada influencia del género sobre la ocurrencia de LES confirman la importancia de factores genéticos y hormonales en la enfermedad. Sin embargo, hay pocas diferencias en las manifestaciones clínicas entre ambos géneros ${ }^{(4)}$. Los afroamericanos e hispánicos son afectados con mayor frecuencia que los caucásicos, presentando además mayor morbilidad ${ }^{(5)}$. El análisis de la tasa de incidencia anual, en 1995, en una población de EE UU, certificó la marcada tendencia por el sexo femenino, dado que la tasa de incidencia anual por 100000 habitantes para LES fue 0,4 para varones blancos versus 3,5 en mujeres blancas, y 0,7 para hombres afroamericanos versus 9,2 para mujeres afroamericanas ${ }^{(6,7)}$; así, el sexo y la raza marcan diferencias en la prevalencia de algunas enfermedades autoinmunes, como el LES ${ }^{(8)}$.

No solo hay evidencias de la relación entre las hormonas sexuales y el desarrollo de LES, sino que también se demuestra el correlato entre la actividad de la enfermedad y los mayores niveles de estrógeno en ambos sexos, así como la menor cuantificación de testosterona. Se sabe además que los andrógenos tienen algunas propiedades inmunosupresoras, por lo que un estado funcional de hipoandrogenismo es importante en la patogénesis de la enfer- 
Tabla 3. Criterios diagnósticos de lupus eritematoso sistémico cumplidos por el paciente.

\begin{tabular}{|c|c|c|}
\hline Criterio & Definición & Comentario \\
\hline Úlceras bucales & $\begin{array}{l}\text { Ulceración bucal o nasofaríngea, usualmente indolora, } \\
\text { observada por el médico }\end{array}$ & $\begin{array}{l}\text { Paciente presentó lesiones en mucosa oral, } \\
\text { no dolorosas. }\end{array}$ \\
\hline Artritis & $\begin{array}{l}\text { Artritis no erosiva que afectaba dos o más articulaciones } \\
\text { periféricas, caracterizadas por sensibilidad dolorosa, } \\
\text { hinchazón o derrame. }\end{array}$ & $\begin{array}{l}\text { Paciente debuta con cuadro de poliartralgias, } \\
\text { presentó artritis no erosiva, con un índice } \\
\text { de artropatía de Jaccoud }>5 \text {. }\end{array}$ \\
\hline Trastornos renales & $\begin{array}{l}\text { Proteinuria persistente mayor de } 0,5 \mathrm{~g} \text { por dia o mayor } \\
\text { de } 3+\text { cualitativo o cilindros celulares; pueden ser } \\
\text { eritrocitos, hemoglobina, cilindros tubulares o mixtos. }\end{array}$ & $\begin{array}{l}\text { Paciente presentó proteinuria de más de } \\
1,7 \mathrm{~g} / 24 \text { horas, además de cilindros } \\
\text { granulosos y leucocitarios persistentemente. }\end{array}$ \\
\hline Trastornos inmunitarios & $\begin{array}{l}\text { Anticuerpos anti-ADN contra ADN natural en títulos } \\
\text { anormales o anti- Smith anticuerpos a antígeno nuclear } \\
\text { Sm o Prueba serológica falsa positiva para sífilis o } \\
\text { Anticuerpo antifosfolipidos positivo (anticardiolipina } \\
\text { IgG o lgM) o anticoagulante lúpico. }\end{array}$ & $\begin{array}{l}\text { Paciente presentó anticuerpos anti-ADN } \\
\text { positivo, con VDRL no reactivo, no se le } \\
\text { realizó anticuerpos anti-Smith ni anticuerpos } \\
\text { antifosfolipídicos. }\end{array}$ \\
\hline Anticuerpos antinucleares & $\begin{array}{l}\text { Título anormal de anticuerpos antinucleares en } \\
\text { inmunofluorescencia o valoración equivalente en } \\
\text { cualquier momento o en ausencia de medicamentos } \\
\text { asociados a lupus inducido por drogas. }\end{array}$ & $\begin{array}{l}\text { Se obtuvo titulo de ANA de } 1: 640 \text {, con } \\
\text { método de inmunofluorescencia, patrón } \\
\text { moteado. }\end{array}$ \\
\hline
\end{tabular}

$\operatorname{medad}^{(9,10)}$. En este sentido, un estudio en varones con LES observó que estos tenían valores significativamente más bajos de testosterona/estradiol que varones sin LES, luego de estimulación con hCG ${ }^{(11)}$. Otro estudio investigó el perfil de hormonas sexuales, como prolactina, testosterona, estradiol, FSH y LH, en varones con LES y controles, observándose que los pacientes con LES tenían significativamente mayores niveles de gonadotropinas (FSH y LH) y $14 \%$ de los pacientes con LES tuvo niveles bajos de testosterona. Estos hallazgos han sido refrendados por otros estudios. Sin embargo, estas observaciones no han sido confirmadas en estudios mayores; además, aún no se ha podido concluir si las anormalidades hormonales son intrínsecas al LES o son consecuencia de cualquier enfermedad crónica no especifica $^{(10,11)}$.

Entre las distintas series de pacientes con LES, la incidencia en varones se encuentra entre 4 y 22\%. En Dinamarca, en 1998, se halló una prevalencia de $11 \%{ }^{(12)}$, mientras en China, en 1999, se encontró una prevalencia de $8 \%{ }^{(13)}$.

Los hallazgos clínicos del LES en varones no difieren diametralmente de lo descrito en mujeres; si bien es cierto que algunos estudios sugieren que el LES es más severo en varones, la diferencia podría ser explicada por el retraso en el diagnóstico o tratamiento en este género, en vista de lo infrecuente que es su consideración inicial dentro de los diagnósticos diferenciales que expliquen el cuadro clínico. Aranow evaluó la severidad de LES en ambos sexos y observó que no hubo diferencias significativas en la severidad de la enfermedad en este rubro. Sin embargo, mostró que cerebritis, fenómeno tromboembólico y anticuerpos antifosfolípidicos son más comunes en hombres ${ }^{(14)}$. Otro estudio comparó características clínicas entre varones y mujeres con LES y concluyó que el lupus eritematoso discoide, papular y nodular mucinoso fueron significativamente más comunes en varones que en mujeres, lo que sugiere que pacientes varones con LES tienden a presentar manifestaciones cutáneas atípicas ${ }^{(15)}$. Un estudio realizado en España. donde 12\% de los pacientes con LES fueron varones, encontró que las lesiones discoides, serositis y lupus eritematoso subcutáneo son más frecuentes en varones que en mujeres ${ }^{(16)}$. Ward y col., en una cohorte de 62 hombres y 299 mujeres. encontró que son más comunes las convulsiones y falla renal por LES en varones que en mujeres ${ }^{(4)}$. Un estudio retrospectivo realizado en 21 varones y 82 mujeres con LES encontró mayor frecuencia de complicaciones tromboembólicas en varones, con lo cual concluyeron que el LES en varones se caracteriza por tener mayor frecuencia y severidad de daño en órgano y una alta prevalencia de complicaciones tromboembólicas potencialmente mortales ${ }^{(17)}$. Otro estudio, realizado en 51 pacientes varones chinos y 201 mujeres con LES, encontró que la prevalencia de daño mayor a órgano no fue diferente entre hombres y mujeres. Sin embargo, hubo mayor porcentaje de pacientes varones con alteración de la función renal y daño cardiovascular ${ }^{(13)}$. En un estudio hindú con 39 pacientes varones lúpicos se observó una mayor incidencia de alteraciones mucocutáneas y falla renal. Sin embargo, a diferencia de otros estudios, concluyó que los pacientes varones tienen una forma menos severa de enfermedad, en comparación con las muje- res ${ }^{(18)}$. Otros estudios retrospectivos en lúpicos varones encuentran que la enfermedad renal $(75 \%)$, rash malar $(70 \%)$ y la artritis (60\%) son las manifestaciones clínicas más frecuentes, mientras que los anticuerpos antinucleares (ANA) (95\%), hipocomplementemia (77\%) y los anticuerpos anti-dsADN (57\%), son los hallazgos de laboratorio más frecuentes. Se vio además un peor pronóstico en pacientes varones con LES ${ }^{(19,20)}$. En otro estudio realizado en 46 varones comparados con 269 mujeres lúpicas, se observó que el derrame pleural, pericarditis, nefritis lúpica, proteinuria, falla renal y anticuerpos anti-Sm fueron más frecuentes en varones ${ }^{(21)}$, con lo cual se sostuvo que el LES en varones se caracteriza por tener manifestaciones clínicas atípicas, severa injuria renal y un peor pronóstico ${ }^{(21,22)}$.

En un estudio hecho en una población similar a la nuestra, donde participaron 107 pacientes varones comparados con 1 209 mujeres latinoamericanas con LES, se observó que los varones tuvieron una mayor prevalencia de nefropatía, trombosis vascular y la presencia de anticuerpos antidsADN; además, concluyeron que existiría mayor severidad del LES en varones que en mujeres ${ }^{(23)}$. En una serie similar hecha en Brasil, en 18 varones y 254 mujeres con LES, se encontró nuevamente que la nefropatia (síndrome nefrótico) fue la manifestación más común en varones, siendo la trombocitopenia y pleuritis también significativamente más frecuentes en esta población. Así como en la mayoría de estudios presentados, también se concluyó que en varones el LES es una enfermedad más severa, con mayor morbilidad, especialmente relacionada a nefropatía ${ }^{(24)}$.

El LES es aún una enfermedad con significativa mortalidad y aunque 5 años después del diagnóstico $92 \%$ de pacientes sobrevive, este pronóstico cae a $82 \%$ de supervivencia a los 10 años, 76\% a los 15 años y solo $68 \%$ a los 20 años. En la mayoría de estudios, los pacientes con enfermedad renal tienen un peor pronóstico. Las causas más comunes de muerte en LES son las infecciones. La actividad del LES contribuye con cerca de un tercio de las muertes tempranas, pero menos comúnmente con muertes tardías. Sin embargo, las muertes relacionadas a enfermedad vascular aguda o crónica, incluyendo 
muerte súbita, son más comunes luego de pasados 5 años del diagnóstico. También, hay una mayor morbilidad asociada con un mayor periodo de supervivencia luego del diagnóstico de LES ${ }^{(25)}$. El efecto del género sobre la supervivencia en pacientes con LES es controversial. En varones con LES se ha encontrado una nefropatía de mayor severidad y una supervivencia más reducida, al compararse con su contraparte femenina. Otros estudios revelan que sí habría diferencia en el daño a órgano y la frecuencia de mortalidad, según género. Un estudio realizado en población china mostró que, aunque la nefritis contribuye en el daño orgánico, no es un determinante mayor en la supervivencia y que las infecciones permanecen como la causa más común de muerte, mientras que el tratamiento con dosis altas de corticoides y la trombocitopenia son considerados como factores de riesgo independientes para mortalidad ${ }^{(26)}$.

En otro estudio, realizado en 300 pacientes, se observó que las neoplasias malignas fueron la causa más común de muerte (20\%), seguidos por infección y enfermedad vascular. La causa de muerte varió dependiendo de la duración de la enfermedad: $50 \%$ de las muertes tempranas fueron por nefropatia, mientras $23 \%$ de las muertes tardías fueron por causas vasculares (27). En otra serie, realizada en 100 pacientes tunecinos, la enfermedad activa (60\%) e infecciones (40\%) fueron las causas más comunes de mortalidad. Las infecciones más frecuentes fueron sepsis bacteriana e infecciones urinarias ${ }^{(28)}$.

Dentro de las referencias nacionales, en una tesis realizada en el Hospital Nacional Cayetano Heredia, entre 1970 y 1990, donde se comparó las historias clínicas de pacientes varones y mujeres con LES, se observó que no hubo diferencias significativas en cuanto a la edad de inicio de la enfermedad, número de criterios al momento del diagnóstico, duración del seguimiento, cuadro clínico, exámenes de laboratorio, actividad extrarrenal, tratamiento y mortalidad. El compromiso articular y renal fueron las manifestaciones clínicas más frecuentes en ambos sexos. En cuanto a la mortalidad, la sepsis fue la causa en la mayoría de los casos ${ }^{(29)}$. Datos similares se encontró en el Hospital Dos de Mayo, con pacientes captados entre 1980 y 1993, observándose además que el rash malar y la fotosensibilidad fueron más frecuentes en mujeres, mientras el derrame articular, úlceras mucosas, lupus discoide, vasculitis cutánea y cuerpos citoides fueron más frecuentes en varones; dentro de las alteraciones de exámenes de laboratorio, la elevación de urea y creatinina fue más frecuente en varones. Las complicaciones más frecuentes en ambos sexos fueron las infecciones, encontrándose la gangrena y ulceraciones por vasculitis más frecuentemente en varones, mientras los efectos colaterales a la quimioterapia fueron más frecuentes en mujeres. La principal causa de muerte en mujeres fue la insuficiencia renal crónica terminal y en varones la infección bronconeumónica ${ }^{(30)}$.

En un estudio realizado en 38 pacientes lúpicos admitidos en UCI del Hospital Rebagliati, Lima, Perú, entre 1998 y 2003, se encontró una mortalidad en UCI del $54 \%$, siendo la causa principal de muerte el shock séptico $(87,5 \%)$, de punto de partida respiratorio ${ }^{(31)}$.

Esta comunicación nos permitió realizar una revisión de la literatura acerca de las características, tanto clínicas como inmunológicas, de una enfermedad poco frecuente en varones, cuyo diagnóstico y tratamiento oportuno mejoran el pronóstico de los pacientes; detallando, así mismo, los hallazgos clínicos divergentes respecto a su presentación en mujeres.

\section{REFERENCIAS BIBLIOGRÁFICAS}

1. James M. Diagnosis of systemic lupus erythematosus. Am Fam Phys. 2003;68(11):6571.

2. Mills A. Systemic lupus erythematosus. N Engl J Med. 1994;330:1871-9.

3. Vilá L, Alarcon G, McGwin G, Friedman A, Baethge $B$, Bastian $\mathrm{H}$, et al. For LUMINA study groups. Early clinical manifestations, disease activity and damage of systemic lupus erythematosus among two distinct US Hispanic subpopulations. Rheumatology. 2004;43:358-63.

4. Ward M, Studenski S. Systemic lupus erythematosus in men: a multivariate analysis of gender differences in clinical manifestations. J Rheumatology. 1990;17(2):220-4.

5. Petri M, Magder L. Classification criteria for systemic lupus erythematosus: a review. Lupus. 2004;13:829-37.

6. Arbuckle R, James J, Dennis G, Rubertone M, McClain M, Kim X. Rapid clinical progression to diagnosis among African-American men with systemic lupus erythematosus. Lupus. 2003;12(2):99-106.

7. McCarty DJ, Manzi S, Medsger TA, Ramsey-Goldman $\mathrm{R}$, LaPorte R, Kwoh C. Incidence of systemic lupus erythematosus. Race and gender differences. Arthritis Rheum. 1995;38:1260-70.
8. Yung Yu C, Whitacre C. Sex, MHC and complement C4 in autoimmune diseases. TRENDS in Immunology. 2004;25(12):78-85.

9. Sequeira J, Keser G. Systemic lupus erythematosus: sex hormones in male patients. Lupus. 1993;2(5):315-7.

10. Mok C, Lau C. Profile of sex hormones in male patients with systemic lupus erythematosus. Lupus. 2000;9(4):252-7..

11. Carrabba M, Giovine C. Abnormalities of sex hormones in men with systemic lupus erythematosus. Clin Rheumatol. 1985;4(4):4205.

12. Voss A, Green A, Junker P. Systemic Lupus erythematosus in Denmark: Clinical and epidemiological characterization of a countybased cohort. Scand J Rheumatol. 1998;27:98105.

13. Mok C, Lau C, Chan T. Clinical characteristics and outcome of southern Chinese males with systemic lupus erythematosus. Lupus. 1999;8(3):18896.

14. Aranow C, Del Guidice J, Barland P, Weinstein A. Systemic lupus erythematosus disease severity in men and women: a case-control study. J Rheumatol. 2002;29(8):1674-7.

15. Kanda N, Tsuchida T, Watanabe T, Tamaki K. Clinical features of systemic lupus erythematosus in men. Characteristics of the cutaneous manifestations. Dermatology. 1996;193(1):6-10.

16. Font J, Cervera R, Navarro M, Pallarés L, LópezSoto A, Vivancos J, Ingelmo M. Systemic lupus erythematosus in men: clinical and immunological characteristics Ann Rheum Dis. 1992;51:10502.

17. Specker C, Becker A, Lakomek HJ, Bach D, Grabensee B. Systemic lupus erythematosus in men--a different prognosis? Rheumatol. 1994;53(6):339-45.

18. Pande N, Malaviya N, Sekharan S. SLE in Indian men: analysis of the clinical and laboratory features with a review of the literature. Lupus. 1994;3(3):181-6.

19. Chang CC, Shih TY, Chu SJ. Lupus in Chinese males: a retrospective study of 61 patients. Lupus. 1995;55(2):143-50.

20. Chang DM, Chang CC, Kuo SY, Chu SJ, Chang ML. The clinical features and prognosis of male lupus in Taiwan. Lupus. 1998;7(7):462-8.

21. You YH, Wu CL. A clinical study on male patients with systemic lupus erythemasus. Lupus. 2000;25(5):499-501.

22. Aydintug A, Domenech I, Cervera.R. Systemic lupus erythematosus in males: analysis of clinical and laboratory features. Lupus. 1992;1 (5):2958.

23. Molina JF, Drenkard C, Molina J, Cardiel MH, Uribe 0 , Anaya JM, et al. Systemic lupus erythematosus in males. A study of 107 Latin American patients. Medicine. 1996;75(3):124-30.

24. Costallat L, Coimbra A. Systemic lupus erythematosus in 18 Brazilian males: clinical 
and laboratory analysis. Clin Rheumatol. 1993;12(4):522-5.

25.Gordon C. Long-term complications of systemic lupus erythematosus. Rheumatology. 2002;41:1095-100.

26. Mocc CC, Lee K. A prospective study of survival and prognostic indicators of SLE in a southern Chinese population. Rheumathology. 2000;39:399-406.

27. Moss K, loannou Y, Sultan S, Haq I, Isenberg D. Outcome of a cohort of 300 patients with systemic lupus erythematosus attending a dedicated clinic for over two decades. Ann Rheum Dis. 2002;61:409-13.
28. Houman M, Smiti-Khanfir M, Ghorbell I, Miled M. Systemic lupus erythematosus in Tunisia: demographic and clinical analysis of 100 patients. Lupus. 2004;13:204-11.

29. Ferrandiz M. Tesis: Estudio de LES en relación al sexo. Lima, Perú: Universidad Peruana Cayetano Heredia; 1991.

30. Albarracín Y. Tesis: LES en el Hospital General 2 de Mayo. Lima, Perú; 1993.

31. Mora C, Segami M. Pronóstico de los pacientes con lupus eritematoso sistémico admitidos a la unidad de cuidados intensivos del HNERM, Lima. Rev. Perú Reuma. 2003;9(2):57-9.
Manuscrito recibido el 13 de febrero de 2008 y aceptado para publicación el 27 de febrero de 2008.

Correspondencia:

Franco Romani Romani

Dirección: Av. Héroes del Pacifico 615. Chorrillos

Lima 9, Perú

Correo-e: frrr812@hotmail.com 